\title{
Difference Spectroscopy in the Analysis of the Effects of Coffee Cherry Processing Variables on the Flavor of Brewed Coffee
}

\author{
Donald J. Lyman, ${ }^{1}$ Robert M. Benck, ${ }^{2}$ and Scott F. Merle ${ }^{2}$ \\ ${ }^{1}$ Department of Bioengineering, University of Utah, Salt Lake City, UT 84112, USA \\ ${ }^{2}$ Batdorf \& Bronson Coffee Roasters, 200 Market Street NE, Olympia, WA 98501, USA
}

Correspondence should be addressed to Donald J. Lyman, dlypr@comcast.net

Received 30 November 2010; Revised 14 February 2011; Accepted 22 March 2011

Academic Editor: A.M. Brouwer

Copyright ( 92011 Donald J. Lyman et al. This is an open access article distributed under the Creative Commons Attribution License, which permits unrestricted use, distribution, and reproduction in any medium, provided the original work is properly cited.

\begin{abstract}
Infrared difference spectroscopy was used to study how changes in the processing of Arabica coffee cherries into green beans affected the flavor of coffee brewed from roasted green beans. Paired samples of green beans, in which the drying step or fermentation/washing step in their processing was altered, were roasted and brewed in a standard manner and their ATR-FTIR spectra obtained. Difference spectra of the 1800 to $1680 \mathrm{~cm}^{-1}$ carbonyl region of water-subtracted spectra of paired samples of these brewed coffees provided data which indicated differences in brewed coffee flavor due to changes in fermentation/washing steps and drying steps involved in the processing of coffee cherries. The role of acid, ketone, aldehyde, ester, lactone, and vinyl ester carbonyl components on the flavor of brewed coffee is proposed that is consistent with the flavors as perceived by the coffee tasters.
\end{abstract}

\section{Introduction}

The blending of taste from volatile and nonvolatile compounds sensed in the mouth and aroma from volatile compounds sensed in the nose gives brewed Arabica coffees their distinct flavor [1-4]. All Arabica coffee cultivars are derived from two original, or heirloom, varieties, the Typica and Bourbon. Mutations and hybrids of Typica from the Amsterdam Botanical Garden and Bourbon from the island of Bourbon are now grown worldwide resulting in significant variation in the flavor profiles of coffee brewed from them. Factors influencing coffee flavor are the species of coffee cultivar, the geographical conditions where the cultivars are grown (including type of soil, climate, and altitude), methods used to process coffee cherries into green beans, and how the green beans are roasted. Processing variables involved in converting the coffee cherries into green beans appears to be of major importance.

In an effort to improve the overall taste of their coffee beans, some coffee plantations are exploring ways to tweak the washing and drying of coffee cherry processing. Normally, determining the effect of these changes rely on taste testing of coffee brewed from roasted green beans. Taste tests are subjective, and a more empirical analytical technique to assess the effect of these variables on the flavor of brewed coffee is of interest, particularly one that sheds light on the molecular changes that produced the perceived flavor differences.

Difference spectroscopy was initially developed to characterize reaction-induced changes in protein conformation by assessing differences between a sample spectrum and a reference spectrum. It was later used to show how changes in processing affected the molecular structure of biopharmaceutical formulations $[5,6]$. It is reasonable, therefore, to look to difference spectroscopy as a method to analyze changes in the molecular composition of brewed coffee resulting from variables in the coffee cherry processing.

In this paper we present the results of a study on coffee brewed from medium roasted Arabica green beans using infrared difference spectroscopy to show the effects of two variables of processing coffee cherries into green beans. The changes in infrared difference spectra were also compared to flavors of brewed coffees reported by a panel of coffee tasters using a standard taste testing method [7]. 


\section{Materials and Methods}

2.1. Roasting of Coffee Beans. Production line bags of green coffee beans were obtained from the Costa Rica La Minita Estate and Nicaragua Los Placeres representing paired samples of green coffee beans in which only the drying method or the coffee cherry washing method was changed. Samples of the green beans were roasted at Batdorf \& Bronson under identical conditions using a Probat BRZ-2 sample roaster (Probat Burns Inc., Memphis, TN, USA). The roasting size was 100 grams of green coffee beans. Each roast was 11 minutes in duration, where the first crack occurred at approx. $8 \mathrm{~min}$ and the roasted beans were dropped at $11 \mathrm{~min}$, just shy of the second crack. The beans were roasted to an Agtron 60 on the M-Basic II color spectrum reader (Agtron Inc., Reno, NV, USA) with a scale from 0 (darkest) to 80 (lightest) color.

2.2. Brewing of Coffee. All roasted beans were ground to a standard cupping grind (similar to that of a French press grind). Coffee was brewed using the standard cupping method, where six ounces of hot $\left(98^{\circ} \mathrm{C}\right)$ filtered water were added to seven grams of ground coffee in a tasting cup and the mixture was allowed to steep for $4 \mathrm{~min}$. The crust floating on the top of each cup was then broken and the coffee aroma noted. The coffee grounds were then stirred to initiate settling, and any residual foam and floating grounds were spooned off.

Prior to the tasting process, samples of each coffee (approx. $25 \mathrm{~mL}$ ) were placed in $120 \mathrm{~mL}$ polypropylene screwcapped jars and refrigerated until analyzed. A sample of the filtered water used in brewing the coffee was also placed in a $120 \mathrm{~mL}$ polypropylene screw-capped jar and refrigerated.

2.3. Taste Testing of Brewed Coffee. The various roasts were tasted and the aroma, acidity, body, and taste noted to describe the flavor of the coffee [7]. The taste testers were Robert Benck, Scott Merle, and three others from Batdorf \& Bronson Coffee Roasters quality assurance group. Five brewings of each coffee were tasted.

2.4. ATR-FT-IR Analyses. Samples of brewed coffee were analyzed using a Thermo Nicolet Nexus 670 FT-IR spectrometer with a liquid nitrogen cooled mercury-cadmiumtelluride (MCT) detector and a Harrick Scientific Prism Liquid ATR cell with a $45^{\circ} \mathrm{ZnSe}$ crystal $\left(\theta=45^{\circ}\right)$. Absorption spectra were obtained from 4000 to $700 \mathrm{~cm}^{-1}$, using 128 scans at a resolution of $4 \mathrm{~cm}^{-1}$ and Norton-Beer medium apodization. Baseline correction, water subtraction, offsetcorrection between 1800 and $1680 \mathrm{~cm}^{-1}$, and other spectral manipulations were done using the Galactic GRAMS/386 program. Spectra of the filtered water used to brew the coffee were used for water subtraction.

Band assignments for the carbonyl absorptions, based on general literature and published spectra of a number of known constituents of coffee, are lactones (1800$\left.1762 \mathrm{~cm}^{-1}\right)$, vinyl esters $\left(1775-1755 \mathrm{~cm}^{-1}\right)$, aliphatic esters $\left(1755-1740 \mathrm{~cm}^{-1}\right)$, aliphatic aldehydes $\left(1739-1724 \mathrm{~cm}^{-1}\right)$, aliphatic ketones $\left(1725-1705 \mathrm{~cm}^{-1}\right)$, aliphatic acids (1714$\left.1700 \mathrm{~cm}^{-1}\right)$, and aromatic acids $\left(1700-1680 \mathrm{~cm}^{-1}\right)[2,8-10]$.

\section{Results and Discussion}

Spectra of aqueous solutions are easily obtained using attenuated total reflection Fourier transform infrared (ATRFT-IR) spectroscopy. The constant high-resolution and highenergy throughput over the entire spectral region and a good signal-to-noise ratio in FT-IR spectroscopy facilitates spectral subtraction of overlapping water absorption bands and enables the examination of the brewed coffee spectra in some detail. Spectra of coffee brewed from medium roasted beans of the various processed green beans were baseline corrected and the water subtracted.

The aroma and taste of brewed coffee result from a complex mixture of simple linear and branched structures to cyclic and heterocyclic structures. A large number of these compounds are acids, ketones, aldehydes, esters, and lactones, including 22 reported to be important compounds in roasted coffee aroma [1-3]. Since the presence of the carbonyl group often appears to confer organoleptic qualities to these compounds, changes in the difference spectrum of various carbonyl groups could be used to characterize changes in coffee flavor resulting from changes in coffee cherry processing.

The reference state spectrum for each paired set was selected and subtracted from the sample spectrum of that set. The resulting difference spectrum was then offsetcorrected between 1800 and $1680 \mathrm{~cm}^{-1}$. Positive peaks indicate increase of carbonyl compound absorptions in the sample, and negative peaks indicate decrease of carbonyl compound absorptions in the sample.

The results of the brewed coffee flavors reported by the taste testing panel were then compared to the infrared difference spectra analysis.

3.1. Comparison of Drying Method on Coffee Cherries from a Single Origin. The La Minita Estate coffee cherries used in this comparison were grown in rich volcanic soil at an altitude of 1200-2000 meters in the Tarrazu region of Costa Rica. The cultivars were a mix of Caturra (a Bourbon varietal) and Typica. Ripe Arabica coffee cherries were processed by a wet method, which involved washing and pulping to remove the cherry skin and some of the pulp, allowing the pulp remnants on the beans to ferment, followed by a second washing. The wet beans were then divided into two batches. One batch was sun-dried, and the other batch was dried mechanically.

With sun-drying, the beans are spread on cement patios with the temperature cycling between 32 and $37^{\circ} \mathrm{C}$ during the day and about $15^{\circ} \mathrm{C}$ during the night. At night the parchment beans are raked into piles and covered. Total drying times are four to eight days to achieve a water content of $14 \%$.

To reduce dependence on weather to dry the wet beans, mechanical drying using rotating drum dryers has also been used. With drum dryers, the temperature is slowly raised to 
about $50-56^{\circ} \mathrm{C}$ and held at that level for most of the heating cycle, then slowly brought back down to room temperature. Total heating time to achieve water content of $14 \%$ is approx. 8 to 10 hrs.

Both sets of dried beans are allowed to rest (30 to 45 days) until the water content is about $12.5 \%$ and then are hulled to remove the outer parchment coating. The resulting green coffee beans are then bagged for shipment to the coffee roaster.

The green coffee beans obtained from these two drying methods were roasted and brewed under identical conditions. Taste testing of the brewed coffees indicated that both mechanical- and sun-drying impart a sweet juicy flavor characterized by orange citrus notes [7]. However, the sundried coffee was heavier bodied with more intensity in its flavor.

Water-subtracted ATR-FT-IR spectra of the 1800 to $1680 \mathrm{~cm}^{-1}$ region of these two coffees are shown in Figure 1. The similarities in the two spectra in their ester (1760-1740 $\mathrm{cm}^{-1}$ ) and aldehyde $\left(1737-1725 \mathrm{~cm}^{-1}\right)$ carbonyl absorptions are what contribute to the citrus notes. The sun-dried coffee shows higher concentrations of ester and aldehyde absorptions, and this is consistent with its more intense flavor. The sun-dried coffee spectrum showed more of the $-\left(\mathrm{CH}_{2}\right)_{4}-$ absorption at $723 \mathrm{~cm}^{-1}$ than the mechanically dried spectrum. Peak height ratio of the $723 \mathrm{~cm}^{-1}$ band to the $1637 \mathrm{~cm}^{-1}$ Amide I band was 3.1 for the sun dried coffee and 2.6 for the mechanically dried coffee, indicating that there is more lipid material in the sun dried coffee. The presence of more lipid (fatty) compounds gives the sensation of the heavier body on the tongue $[3,7,11]$.

To more quantitatively determine the drying method effect on the flavor of the two coffees, the difference spectrum was obtained using the mechanically dried coffee as the reference spectrum. Water was subtracted from all spectra. The spectrum of the mechanically dried coffee was subtracted from the spectrum of the sun-dried coffee. This difference spectrum is shown in Figure 2. The compounds that are present in higher concentrations in the sun dried coffee are indicated by the absorptions that are above the zero line of the $Y$-axis. The compounds that are present in lower concentrations are indicated by any absorption that appear below the zero line of the $Y$-axis.

Although the sun-dried coffee spectrum showed more of the $-\left(\mathrm{CH}_{2}\right)_{4}$ - absorptions at $723 \mathrm{~cm}^{-1}$ than $\operatorname{did}$ the mechanically dried coffee, the difference spectrum showed that it had lower aliphatic acid carbonyl content than did the lighter bodied mechanically dried coffee. This suggests that the lipids that give the sun-dried coffee a heavier body are lipid esters (1739-1744 $\mathrm{cm}^{-1}$ ) and not lipid (fatty) acids.

The larger concentration of aldehyde and ester components in the sun-dried coffee in the difference spectrum is consistent with the greater intensity of flavor in the sun-dried coffee.

While the difference spectrum indicates that mechanically dried coffee has more vinyl esters and lactone carbonyls absorbing around 1792, 1780, and $1773 \mathrm{~cm}^{-1}$ than sun-dried coffee, they do not appear to be of sufficient concentration to alter the more intense flavor due to the aldehydes and esters.

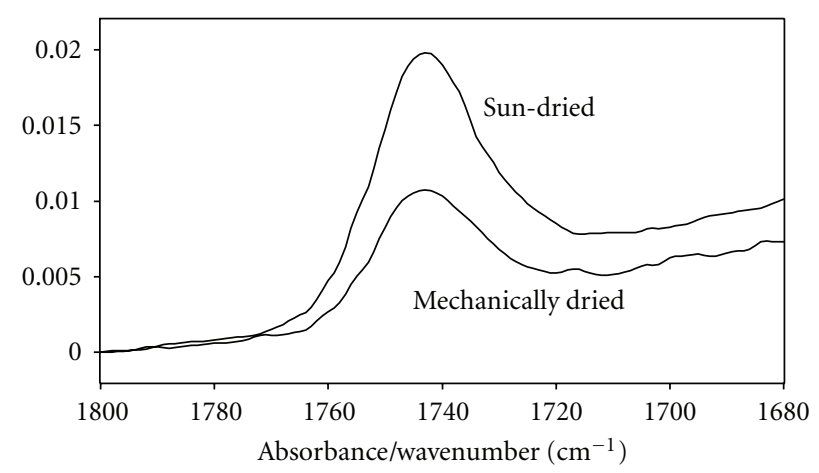

Figure 1: ATR water-subtracted spectra of coffee brewed from roasted sun-dried La Minita beans and mechanically dried La Minita beans.

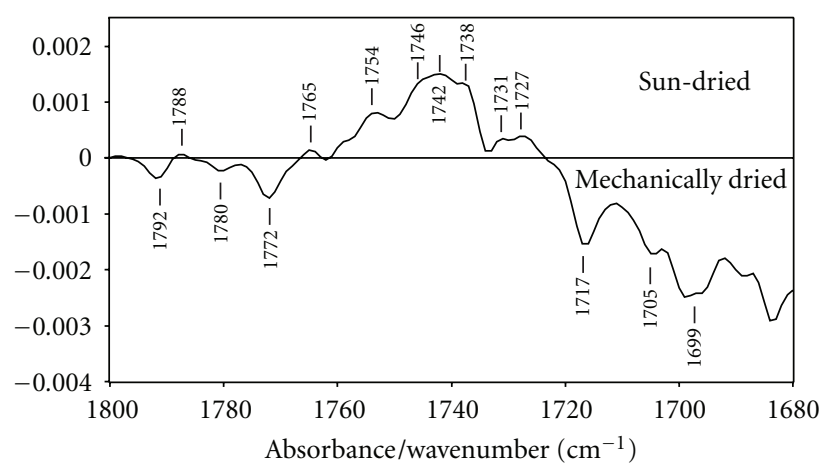

FIGURE 2: Difference spectrum of brewed coffee: water-subtracted spectrum of brewed coffee from sun-dried La Minita beans minus water-subtracted spectrum of brewed coffee from mechanically dried La Minita beans.

These differences in the composition of the two brewed coffees are most likely attributable to exposure to the sun's radiation of the pulp remnant left on the beans during the drying process, which results in increased amounts of adsorbed and absorbed materials. On roasting, these materials give rise to increased amounts of aldehydes and esters.

It is interesting to note the flavor changes that were observed when both lots of green beans had aged for a year. When these aged beans were roasted in a manner similar to fresh green beans, the flavor of coffee brewed from the old sun dried beans retained a heavy body and maple sweetness with an orange-like acidity. In contrast, the flavor of coffee brewed from the old mechanically dried beans had noticeably changed, though still full bodied and sweet. It had a subdued acidity, distinct woody notes, and a dry, cloying aftertaste. The aging process involves oxidation and enzymatic degradation of some of the molecular constituents of green beans, especially the unsaturated molecules. Thus, this would alter the types and relative concentrations of the various compounds formed by the Maillard and Strecker degradation reactions during the roasting process. The fresh mechanically dried beans initially showed lower concentrations of esters and aldehydes compared to the fresh, 
sun-dried coffee (see Figure 1); it is therefore reasonable to expect that aging of the mechanically dried beans would result in reducing the concentration of aldehyde constituents further. This would reduce their effect of masking the contributions of ketones, lactones, and vinyl esters to coffee flavor, thus allowing the woody tones in the brewed coffee to be noted.

3.2. Comparison of Fermentation Effects on Coffee Beans from a Single Origin. The Los Placeres Estates coffee cherries used in this comparison of fermentation effects were grown at an altitude between 900 and 1200 meters in the Matagalpa region of Nicaragua. The cultivars are a mix of Caturra $(65 \%)$, Catuai (15\%), Maragogype (10\%), and Bourbon $(10 \%)$. The harvested coffee cherries were processed using two washing methods, potentially altering the type and amount of fermentation products residing on the depulped cherries.

In the standard washed process, coffee cherries were depulped with water, then allowed to ferment for about 36 hrs in concrete tanks. The fermented depulped cherries were then passed to a second washing channel, where any residual mucilage was actively washed off. The coffee beans were then spread on drying patios and sun-dried. The dried coffee beans produced by the washed process are deep jade in color.

In the pulp-natural process, coffee cherries were depulped with water and then immediately spread on drying patios to sun-dry. All fermentation of the residual mucilage occurs in the presence of the sun's radiation, and any adsorbed and absorbed fermentation material remains on the dried beans. Therefore, the chemistry of fermentation will likely be different between the pulp-natural and washed cherries. In addition, the concentration of some of these products will be higher in the pulp-natural process since the fermented beans are not washed a second time. The dried pulp-natural beans are somewhat yellower in appearance. The dry ( $12.5 \%$ water) beans from both processes are hulled to remove the outer parchment, then bagged and sent to coffee roasters.

Green coffee beans from the washed and pulp-natural processes were roasted and brewed in a similar manner for taste testing [7].

The washed coffee had clean vibrant acidity, medium body, sweet taste (chocolate sweetness), and a crisp clean finish. There were notes of cocoa powder and chocolate malt. In contrast, the pulp-natural coffee was heavy bodied with a lingering fruity sweetness. Darker fruit notes, specifically plum, were present. The acidity was muted in comparison to the washed coffee.

Water-subtracted ATR-FT-IR spectra of the 1800 to $1680 \mathrm{~cm}^{-1}$ region of these two coffees (Figure 3) show distinctly different absorptions, with the washed coffee showing increased absorptions of specific lactones around 1792 and $1772 \mathrm{~cm}^{-1}$, ketones at $1717 \mathrm{~cm}^{-1}$, and aliphatic acids around $1705-1699 \mathrm{~cm}^{-1}$. This is not unexpected since the second active washing step of the washed process would be expected to remove some materials formed in the fermentation step. One would also expect that the radiation

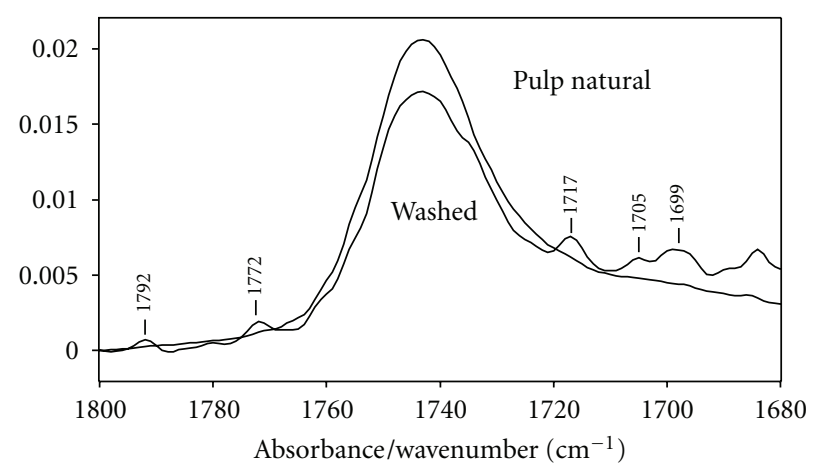

FIGURE 3: ATR water-subtracted spectra of coffee brewed from roasted pulp-natural Placeres beans and washed Placeres beans.

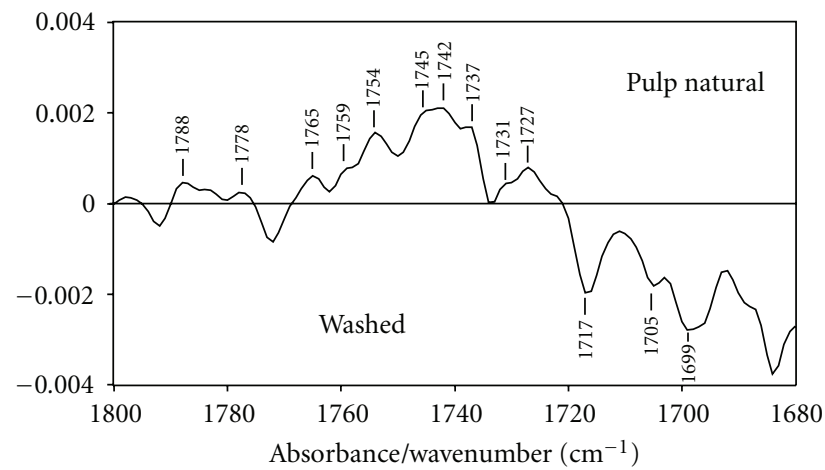

FIGURE 4: Difference spectrum of brewed coffee: water-subtracted spectrum of brewed coffee from pulp-natural Placeres beans minus water-subtracted spectrum of brewed coffee from washed Placeres beans.

effect of the sun on the residual mucilage on the pulpnatural bean would result in different materials being formed during its fermentation step. The FSD and 2nd derivative spectra also indicated differences in both composition and concentration of carbonyl compounds in these two brewed coffees. While the washed coffee had about 39 different carbonyl compounds, the pulp-natural coffee had about 44 different carbonyl compounds.

To more quantitatively determine the effect of changes in fermentation on the flavor of brewed coffee from these beans, the washed coffee was chosen as the reference spectrum. The difference spectrum obtained by subtracting the washed coffee spectrum from the pulp-natural coffee spectrum is shown in Figure 4. Compounds that are present in higher concentrations in the pulp-natural coffee are indicated by the absorptions that are above the zero line of the $y$-axis. Compounds that are present in lower concentrations in the pulp-natural coffee are indicated by absorptions that appear below the zero line of the $y$-axis.

Again, the combination of ester absorption at $1742 \mathrm{~cm}^{-1}$ in the difference spectrum and increased $-\left(\mathrm{CH}_{2}\right)_{4}-$ absorption at $723 \mathrm{~cm}^{-1}$ in the water-subtracted spectrum indicates more lipid (fatty) ester material in the pulp-natural brewed coffee. The peak height ratio of the $723 \mathrm{~cm}^{-1}$ band to the $1637 \mathrm{~cm}^{-1}$ Amide I band was 10.1 compared to 3.7 for 
the washed coffee. This would result in the sensation of heavier body, intensity, and lingering taste in the pulp natural brewed coffee. The pulp-natural coffee also has a more complex mixture of vinyl ester, lactone, ester, and aldehyde components that contribute to the dark fruit notes.

In contrast, the washed coffee has more aliphatic acid carbonyl (1714-1699 $\mathrm{cm}^{-1}$ ) suggesting more of other acidic material. The chocolate notes are most likely related to these aliphatic acid and ketone components. While it also has more of certain lactone and vinyl ester $\left(1792\right.$ and $\left.1772 \mathrm{~cm}^{-1}\right)$ components than the pulp-natural, these do not seem to contribute to any particular floral or fruit notes to the brewed washed coffee.

Even though the coffee cherries underwent a more active fermentation step in the washed process, it appears that the second washing step removes, or at least reduces, the concentration of some of these fermentation by-products. It is also likely that in the pulp-natural process, the sun's radiation in the fermentation/drying step resulted in more materials being formed from the residual mucilage, which produced more fruity organoleptic carbonyl compounds in the roasted coffee beans.

Thus, the method of fermentation and any postfermentation washing steps can have a significant effect on the taste of brewed coffee. Sun radiation during fermentation appears to be an important contributor to the fruity flavor of brewed coffee.

\section{Conclusion}

Difference spectroscopy of paired samples of brewed coffee is useful in studying compositional changes in brewed coffee due to variations in processing coffee cherries into green beans. The 1800 to $1680 \mathrm{~cm}^{-1}$ carbonyl region of ATR-FTIR difference spectra provides important data on the general composition of many organoleptic vinyl esters, lactones, esters, aldehydes, ketones, and acids present in the brewed coffee, and these data appear to correlate well with the taste and aroma perceived by the coffee tasting panel. Changes in the fermentation step in processing coffee cherries to green beans result in more significant differences in the flavor of coffee than changes in the drying steps. Exposure to the sun's radiation during both the fermentation and the drying steps appears to be an important factor, producing more fruity flavor in brewed coffee.

The presence of lipid (fatty) esters contribute to a heavier body and lingering taste on the tongue. Higher concentrations of a larger number of ketone, aldehyde, ester, lactone, and vinyl ester constituents contribute to the intensity of flavor. This is particularly true of ester and aldehyde compounds. Variations in the types and concentrations of these compounds determine whether the flavor is sweeter and fruitier from higher concentrations of esters or more floral and spicy from higher concentrations of aldehydes. Lactones and vinyl esters contribute to woody and pungent flavors. Chocolate flavors appear to be imparted by acids and ketones.

\section{Acknowledgments}

The authors thank the owners of the La Minita Estate (Costa Rica) and the Los Placeres Estate (Nicaragua) for their helpful discussions of their coffee cultivars and processing methods. They thank the coffee tasters from Batdorf \& Bronson for their flavor descriptions of the four brewed coffees used in this study.

\section{References}

[1] R. J. Clarke and R. Macrae, Eds., Coffee: Vol. 1. Chemistry, Elsevier, New York, ny, usa, 1985.

[2] I. Flament, Coffee Flavor Chemistry, John Wiley \& Sons, New York, NY, USA, 2002.

[3] A. Illy and R. Viani, Eds., Espresso Coffee, The Chemistry of Quality, Academic Press, New York, NY, USA, 1995.

[4] T. H. Parliament and H. D. Stahl, "What makes that coffee smell so good?” Chemtech, vol. 25, pp. 38-49, 1995.

[5] A. Barth and C. Zscherp, "What vibrations tell us about proteins," Quarterly Reviews of Biophysics, vol. 35, no. 4, pp. 369-430, 2002.

[6] J. S. Vrettos, R. P. Affleck, J. Guo, T. M. Spitznagel, and R. Krishnamurthy, "Application of difference spectroscopy to biopharmaceutical formulation development," American Biotechnology Laboratory, vol. 24, no. 6, pp. 24-27, 2006.

[7] T. R. Lingle, The Coffee Cuppers Handbook: A Systematic Guide to the Sensory Evaluation of Coffee Flavor, Specialty Coffee Assoc. of America, Washington, DC, USA, 2nd edition, 1992.

[8] L. J. Bellamy, The Infrared Spectra of Complex Molecules, vol. 1, Chapman \& Hall, London, UK, 3rd edition, 1975.

[9] G. Socrates, Infrared Characteristic Group Frequencies, John Wiley \& Sons, New York, NY, USA, 2nd edition, 1994.

[10] R. J. Keller, The Sigma Library of FT-IR Spectra, vol. 1, 2, Sigma Chemical Co., St. Louis, Mo, USA, 1st edition, 1986.

[11] E. Guichard, M. Fabre, and P. Relkin, "Flavor release from food emulsions varying in their composition in fat and proteins and its effect on flavor perception," American Laboratory, vol. 40, no. 3, 2008. 


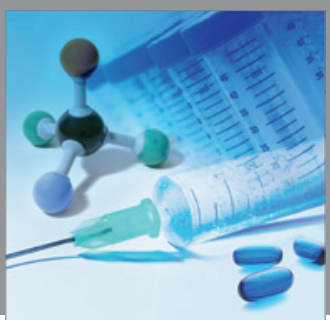

International Journal of

Medicinal Chemistry

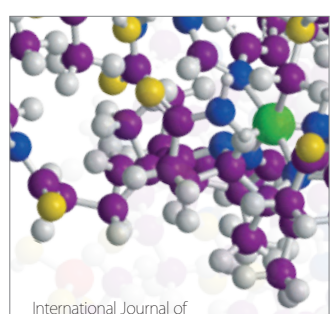

Carbohydrate Chemistry

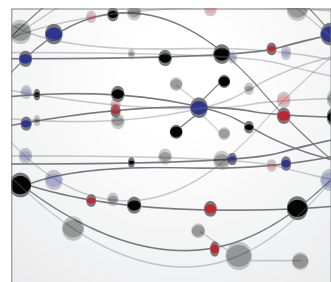

The Scientific World Journal
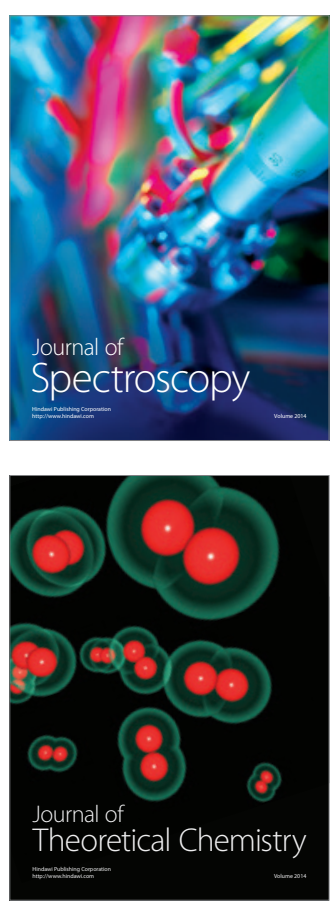
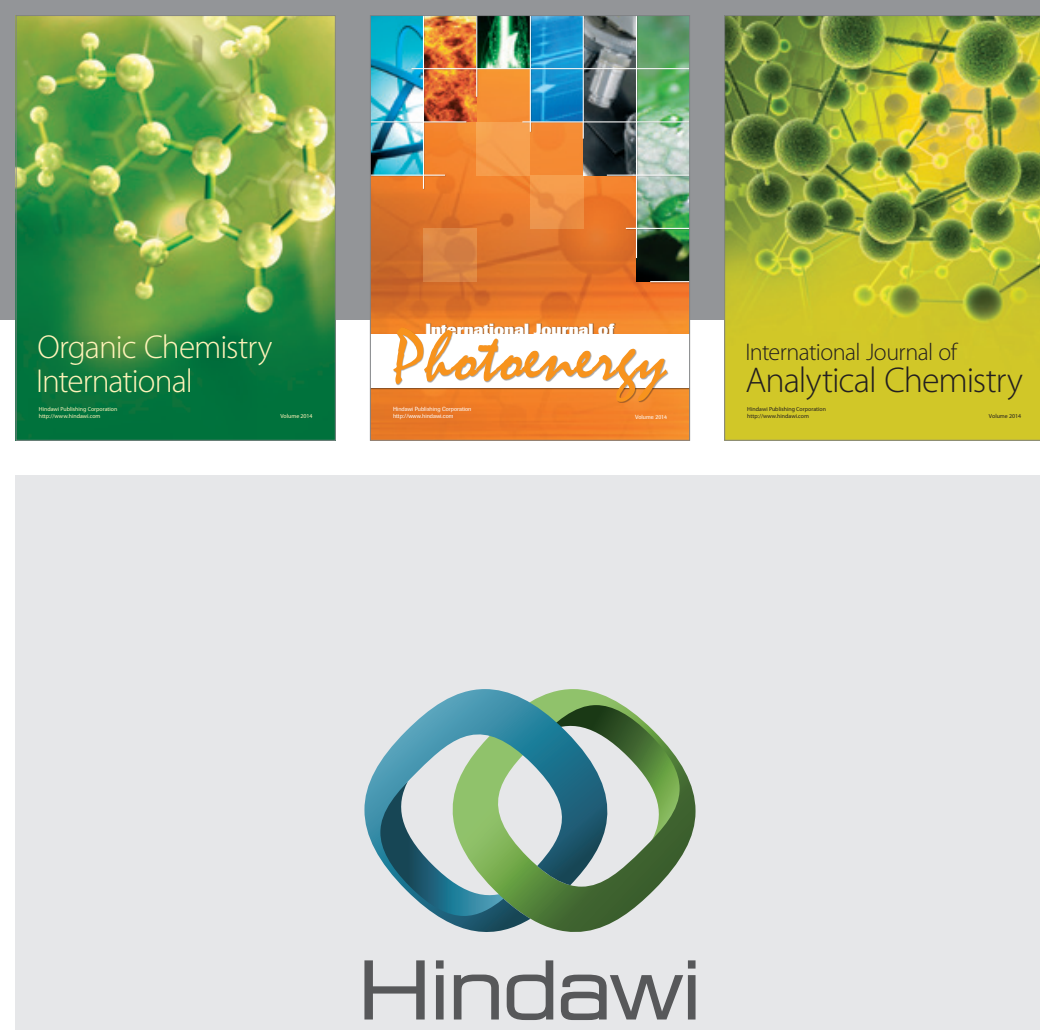

Submit your manuscripts at

http://www.hindawi.com
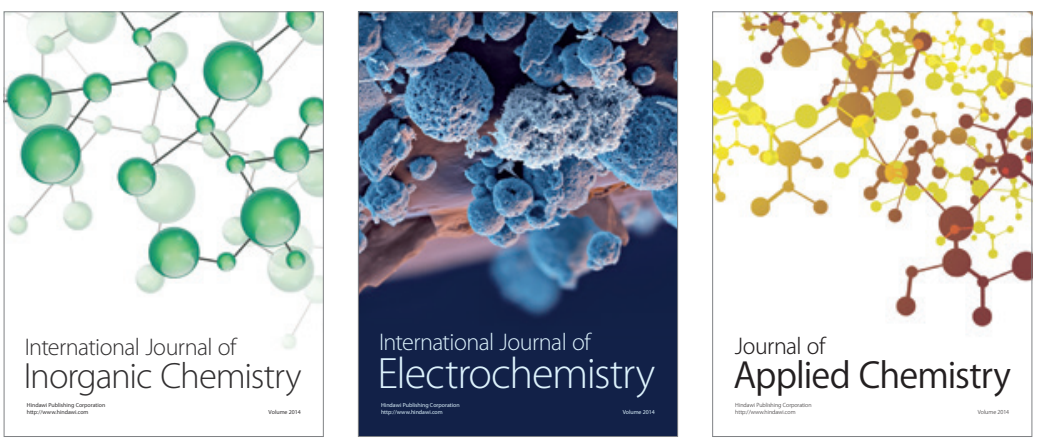

Journal of

Applied Chemistry
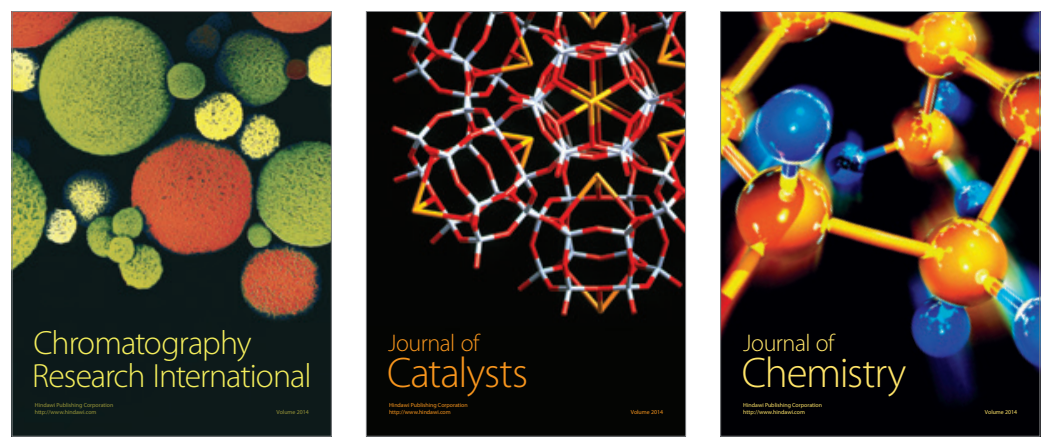
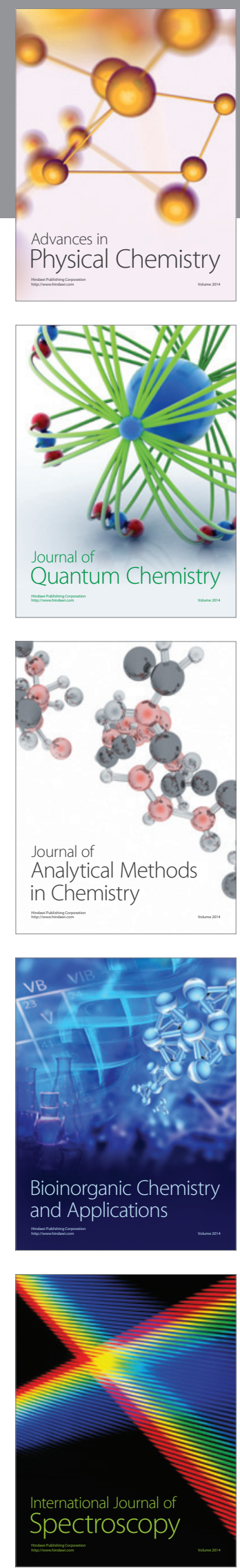